\title{
INTERGRANULAR CRACK NUCLEI IN POLYCRYSTALLINE ALUMINA ${ }^{1}$
}

\author{
M.A. Aswad ${ }^{1}$ and T.J. Marrow ${ }^{2}$ \\ ${ }^{1}$ School of Materials, The University of Manchester, Manchester, M13 9PL, UK \\ ${ }^{2}$ Department of Materials, The University of Oxford, Oxford, OX1 3PH, UK
}

\section{KEYWORDS}

Crack Nucleation, Alumina, Digital Image Correlation, Intergranular Thermal Stress

\begin{abstract}
Digital image correlation has been applied to optical images in order to detect crack nuclei in fine-grain size pure and $\mathrm{Cr}$-doped aluminas. Individual intergranular crack nuclei within areas comprising tens of thousands of grains were detectable, and their crack lengths and opening strains obtained as a function of load prior to unstable fracture. Identification of the crack nuclei by this method allowed the grain boundary plane and grain orientations in the vicinity of crack nuclei to be characterised by electron microscopy. Crack nuclei were found to develop at boundaries that are predicted to have higher tensile thermal strains, caused by the orientation of the grain boundary plane relative to the adjacent grains.
\end{abstract}

\section{INTRODUCTION}

Fracture of ceramics invariably originates from some form of pre-existing defect [1]. Such defects may result from processing (e.g. pores or inclusions) or they may be microcracks at grain boundaries. In polycrystalline ceramics, anisotropy of the crystal thermal expansion coefficients causes internal residual stresses to develop on cooling from the fabrication temperature. These residual stresses can result in spontaneous cracking, primarily along grain boundaries [2]. This is shown by the clear effect of grain size on the fracture strength of alumina [3], for example. Processing may also influence the distribution of grain orientations and grain boundary structures in ceramics [2], and it is of interest to understand whether this may have a significant effect on the development of intrinsic defects and

\footnotetext{
${ }^{1}$ NOTICE: this is the author's version of a work that was accepted for publication in Engineering Fracture Mechanics. Changes resulting from the publishing process, such as peer review, editing, corrections, structural formatting, and other quality control mechanisms may not be reflected in this document. Changes may have been made to this work since it was submitted for publication. A definitive version was subsequently published in Engineering Fracture Mechanics, Volume 95, November 2012, Pages 29 - 36 (http://dx.doi.org/10.1016/j.engfracmech.2012.08.005)
} 
strength. The aim of this work is to develop a better understanding of the relationship between microstructure and the crack nuclei. This requires direct observation of the population of significant defects in the microstructure; a novel technique for this is demonstrated in this paper.

Digital Image Correlation (DIC) allows full field displacement measurements to be obtained throughout the deformation of a material [4]. DIC operates through the discretisation of an image into multiple areas (i.e. "interrogation windows"), which are correlated with the same windows in following images. Displacement vectors can be obtained for the change in position of each interrogation window, allowing strain distributions across the full image to be calculated [5]. DIC allows sub-pixel displacement measurements [6], and is independent of scale; the measurement depends on the image capture camera and in particular its bit depth and resolution. Displacement vector accuracy is dependent on the interrogation window size employed and while larger windows provide for more accurate measurements, they decrease the spatial resolution of the maps of displacement and strain. The achievable accuracy is around 0.01 pixels for an interrogation window size exceeding $128 \times 128$ pixels, decreasing to the order of 0.1 pixels for a $16 \times 16$ pixel window size [7].

Conventional microscopy cannot always visualise defects, as it generally relies on the contrast difference between the open crack and the adjacent solid material. This can be negligible in tightly closed or narrow cracks, presenting significant limitations for optical microscopy. Higher resolution techniques, however, such as scanning electron microscopy can have restricted areas of observation. Novel techniques, such as scanning acoustic microscopy [8], have been used previously to visualise such small crack openings in alumina, but this is not suitable for the in-situ study of crack nucleation. Digital image correlation has been found to be suitable for the in-situ study of tight crack nuclei. Its sensitivity allows the detection of cracks by measurement of their opening displacements using optical microscopy, whilst observing a relatively large area that can be representative of the sample. Recent examples of the application of digital image correlation include studies of stress corrosion crack nucleation in ambient temperature liquid environments [9] and also high temperature water [10], slow atmospheric stress corrosion cracking initiating from surface salt deposits [11] and also brittle crack nucleation and growth in nuclear graphite [12, 13]. This paper reports its first application to cracking in a ceramic, such as alumina.

\section{EXPERIMENTAL DETAILS}

The objective was to observe crack nuclei in pure alumina (99.8\%) and Cr-doped alumina $(99.8 \%+0.1 \% \mathrm{Cr})$ microstructures ${ }^{2}$ with average grain size of $1.5 \mu \mathrm{m}$ and $3.6 \mu \mathrm{m}$ respectively. Four point bend fracture tests were conducted under displacement control, with samples of nominal dimensions of $48 \times 10 \times 2 \mathrm{~mm}$. Digital image correlation (DIC) was used to observe the surface displacements and thereby nominal strains (i.e. due to cracking and damage development) in the microstructure.

The test setup for observation under flexural loading is shown in Figure 1 . The edges of the samples were polished to remove cutting damage, which would reduce sample strength. The outer and inner spans were $22 \mathrm{~mm}$ and $2.5 \mathrm{~mm}$. Steel rolling pins were used to minimize contact friction, and were self-aligned to the sample using a ball bearing mount. Significant contact damage was not expected because of the high compressive strength of alumina. The loading stage was mounted on a high precision $X-Y$ stage (Thorlabs), with a 2-

\footnotetext{
${ }^{2}$ Samples provided by Almath Crucibles Ltd, Newmarket, UK
} 
axis tilt capability to ensure the observed surface remained orthogonal to the camera axis. A strain gauge was attached to the upper surface of the sample, adjacent to the observed region, to measure the applied strain. The displacement was incremented by intervals of $0.02 \mathrm{~mm}$ (equivalent to an elastic surface strain increment of approximately 0.0001 ) at 60 minute intervals. A 12 bit Image Pro X CCD digital camera $(2048 \times 2048$ pixels) was used to record images at each of 25 overlapping locations at each increment, covering a total area of approximately $(500 \times 500 \mu \mathrm{m})$. For each observation, 20 images were averaged to remove transient effects such as vibration or illumination fluctuations. An oil immersion lens with x100 magnification was used, with a working distance of $0.25 \mathrm{~mm}$ and immersion oil (Fractoil) of 1.517 refractive index [14]. The field of view of each image was approximately $100 \times 100$ $\mu \mathrm{m}$. Differences in the projection of the field of view on the CCD array between experiments meant that the resolutions were $0.085 \mu \mathrm{m} /$ pixel and $0.046 \mu \mathrm{m} /$ pixel for the pure alumina and Cr-doped alumina respectively.
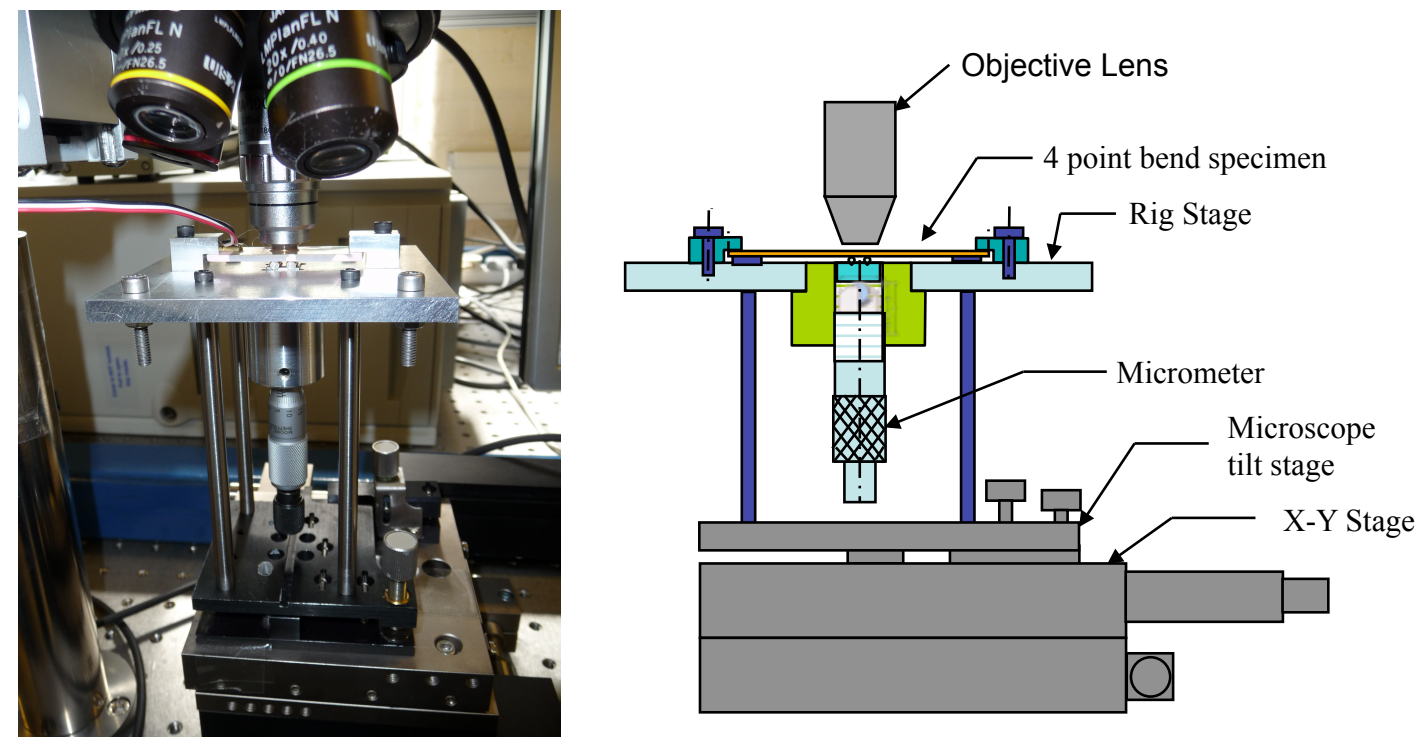

Figure 1: a) Rig for in-situ optical observation of the tensile surface during four point bend loading. b) Schematic illustration of experimental set.

A surface with suitable random features for DIC analysis was achieved by thermal etching at $1400^{\circ} \mathrm{C}$ in air for 10 hours and then gold coating for optical reflectivity with an Edwards S150B sputter coater using an exposure time of 3 minutes (Figure 2). Images were analysed using the LAVision Davis Image Correlation software (version 7.2). An interrogation window size of $64 \times 64$ pixels, with a $50 \%$ overlap was selected for analysis of the pure alumina, and $128 \times 128$ pixels with a $50 \%$ overlap for the Cr-doped alumina. These were found to be the optimal parameters since the image feature density (i.e. the heterogeneity of contrast) within the grains for the Cr-doped alumina structure was lower than for pure alumina after etching. 

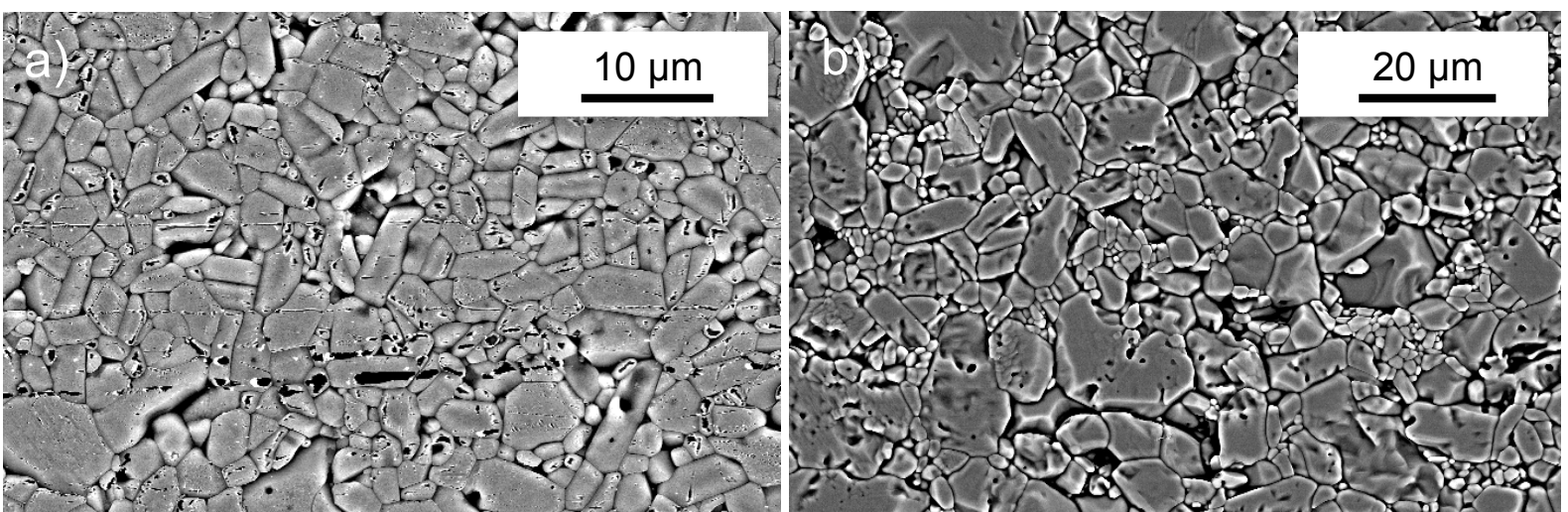

Figure 2: Microstructures prepared for digital image correlation (thermally etched and gold sputtered), and observed by scanning electron microscopy, a) pure alumina and b) Cr-doped alumina.

\section{RESULTS AND DISCUSSION}

Image correlation of observations at different levels of applied load reveals cracks; differentiation of the displacement field (parallel to the loading direction) gives features of high, localised, apparent strain (Figure 3a), which can be used to visualise the opening displacements of cracks. Comparison of the locations of these strain features with the microstructure image from which they are derived (Figure $3 b$ and $3 c$ ) shows them to be coincident with grain boundaries, indicating that they are intergranular cracks. The strain features were not associated with porosity, and appear along boundaries that tend to be perpendicular to the applied stress. Cracking at these boundaries cannot be detected without the use of DIC analysis.

a)

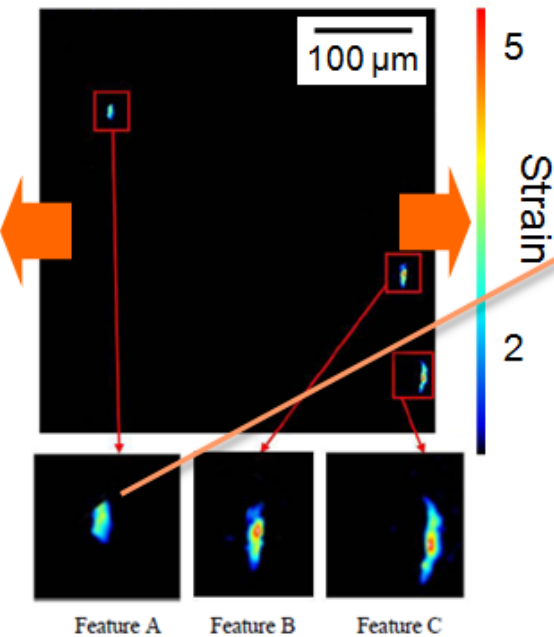

b)

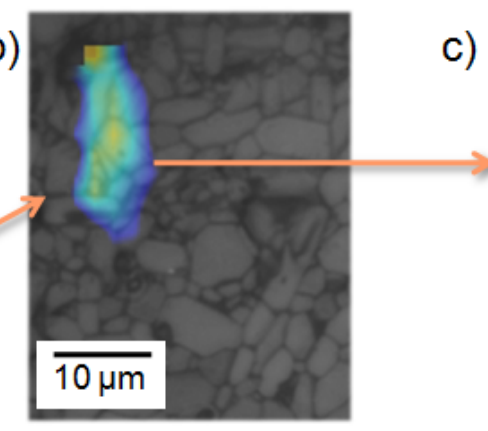

c)

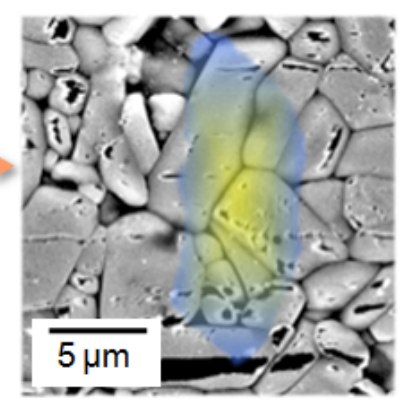

Figure 3: Example of the identification of strain features by in- situ observation (pure alumina), a) DIC strain map (the scale is in nominal units) with larger strain features identified, b) optical image with strain feature (A) superposed at its measured location, c) SEM image of the same region with strain 
feature (A) from the DIC analysis of the optical images superposed. In all cases, the displacements parallel to the loading direction are used to calculate strain.

The magnitude of the strain features is very significant compared to the average background strains measured by DIC (Figure 4). Comparison between the strain gauge output and DIC measurement of average background strains found that the strain measured by DIC was about half $(\sim 54 \%$ for both materials) that measured by the strain gauge, which had been shown by independent experiments to be reliable. This indicates that the DIC measurements of displacement in these experiments should be regarded only as qualitative; the heterogeneity of contrast in the images was insufficient for accurate measurement.

A threshold is used to segment the strain map, relative to the background noise, to identify and label these strain features. This allows the development of their maximum strain, and hence their crack opening displacements, to be tracked with increasing applied strain. Although the data are noisy and the values should be regarded as qualitative, Figure 4 shows a general trend of increasing feature strain ("Feature Exx") with increasing applied strain, as would be expected for a crack that is opening in response to applied stress. The visualisation of cracks by this method enables their surface crack length to be estimated; the effect of the segmentation threshold on the measured distribution of feature lengths is shown in Figure 5 (the measurement uncertainty arises from the chosen DIC analysis window size and overlap). The population of feature lengths is relatively stable with applied strain, particularly for larger features, indicating that the observed cracks are generally stable. Examples for single features are shown in Figure 6; the observed increase in feature length with applied strain in Figure 6(a) does suggest some stable crack growth may occur, perhaps through coalescence, whereas that in Figure 6(b) may be an artifact of the measured strains being relatively close to the segmentation threshold. This might be studied in future by higher resolution observations, which would require image correlation analysis of scanning electron microscopy at selected locations with identified cracks and also alternative patterning techniques.
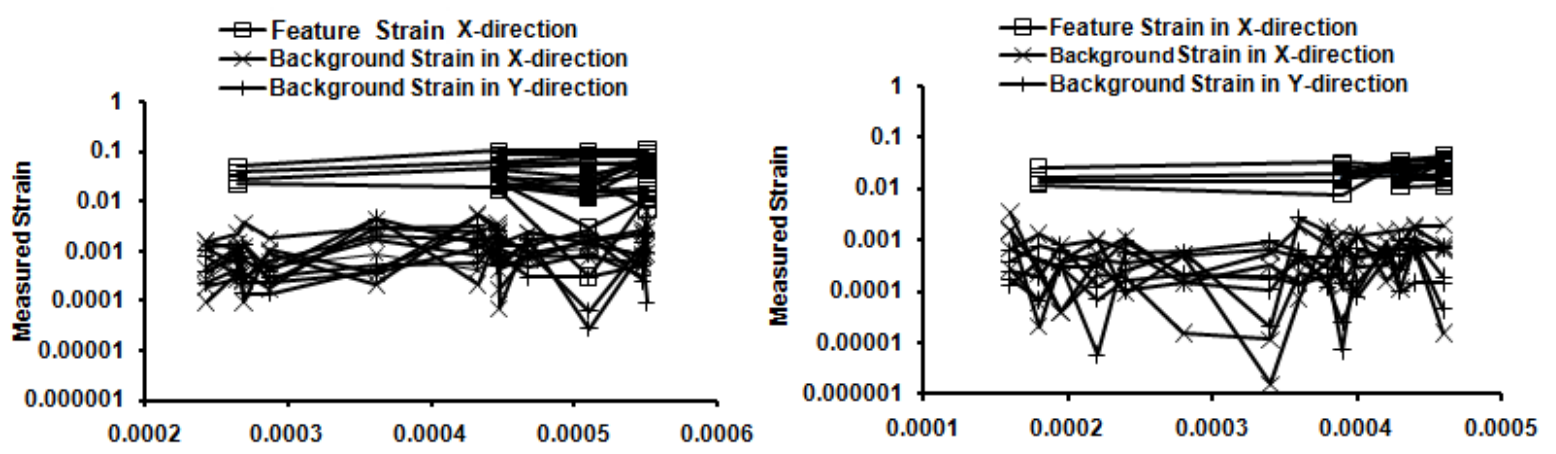

a)

Applied Strain
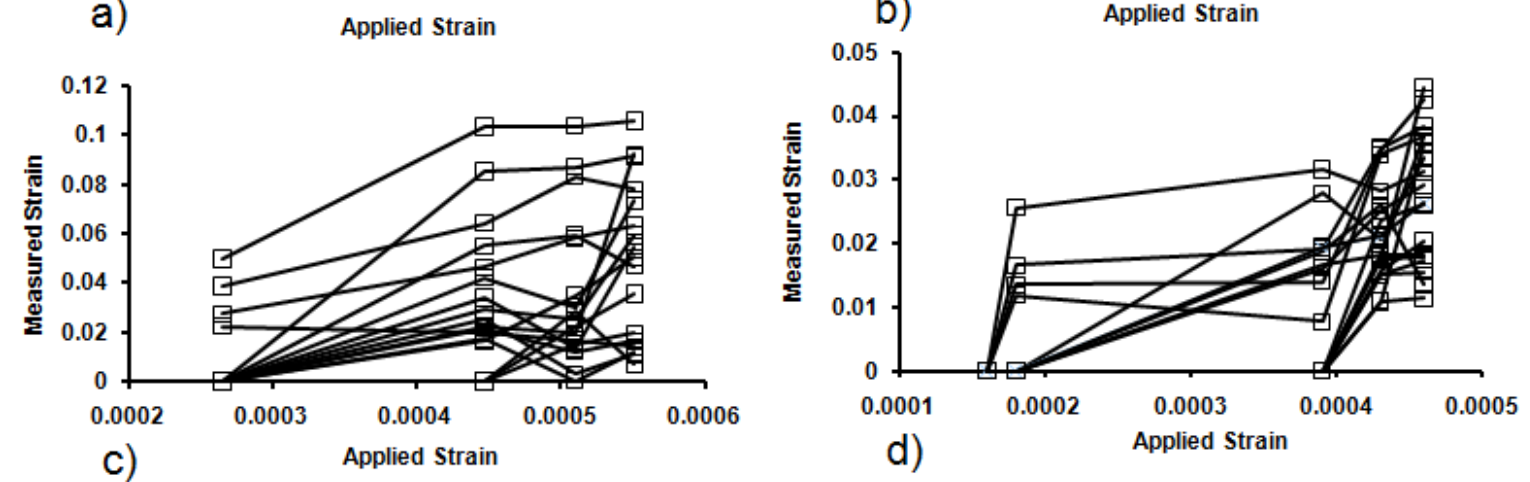

Figure 4: Effect of applied strain on the measured strain for significant strain features and the background strains as a function of applied strain for a, c) pure alumina and b, d) Cr-doped alumina. 
(Exx is parallel to the applied bending stress, and Eyy is perpendicular). The background strain is measured in areas with no apparent localised strains. $(a, b)$ in Log scale and (c, d) in normal scale.
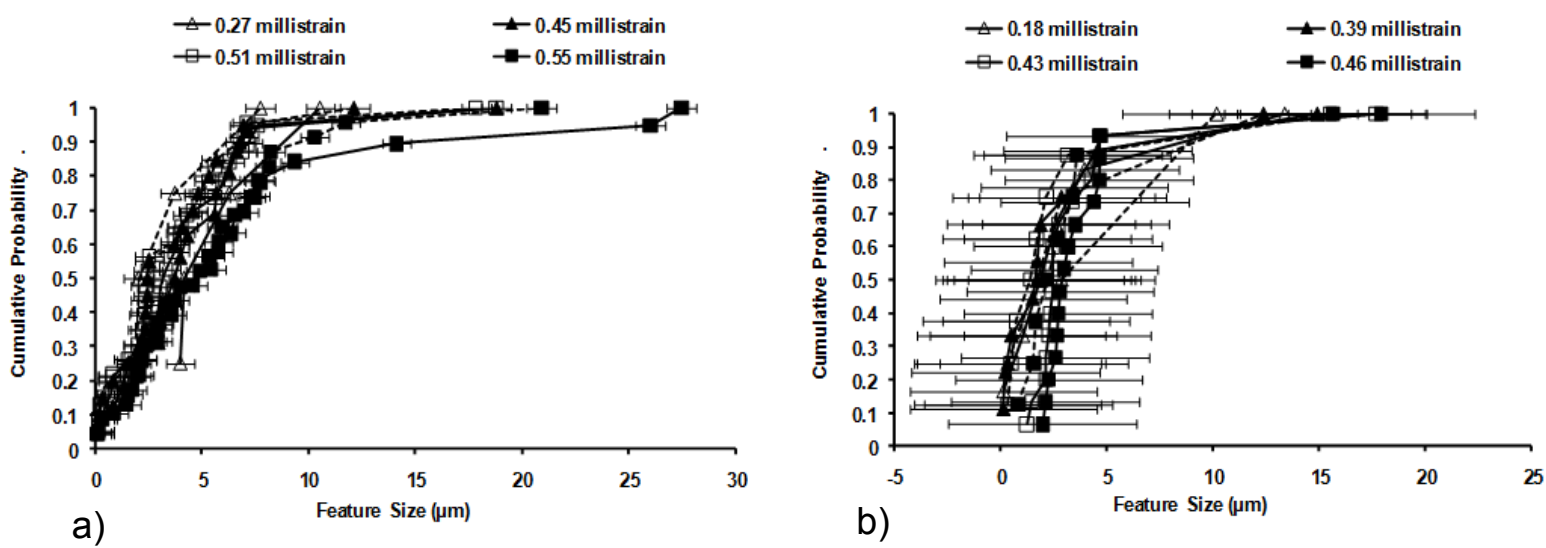

Figure 5: Relationship between the strain feature length and cumulative probability of observation for a) pure alumina, b) Cr-doped alumina. Legend describes the applied strain at which the features were measured. Solid lines show data for a segmentation threshold of 0.002 (2 millistrain), and dotted lines show data for a higher segmentation threshold of 0.005 (5 millistrain).

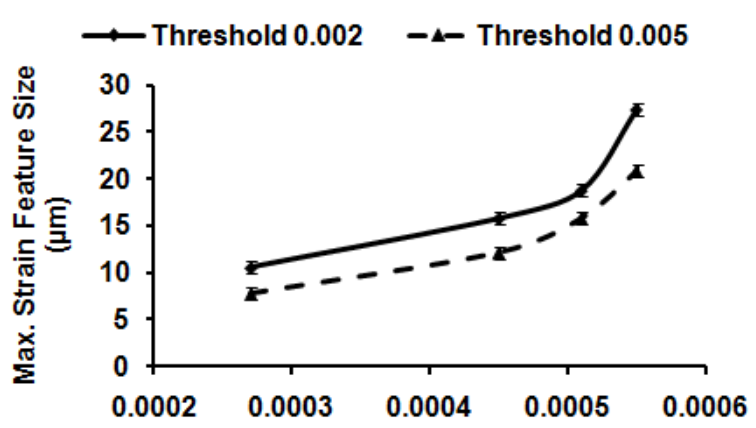

a)

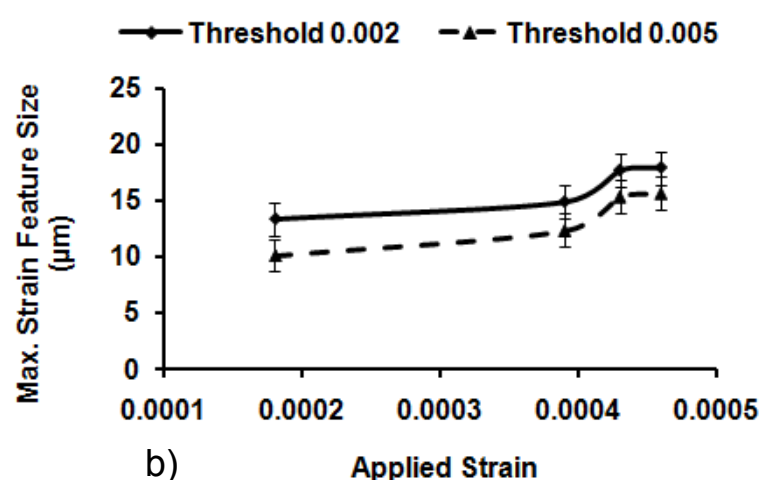

b)

Figure 6: Examples of the rffect of segmentation threshold on the maximum strain feature size as a function of the applied strain for a) pure alumina and b) Cr-doped alumina.

Having identified crack nuclei through the use of digital image correlation, the grain boundaries associated with these were examined. The objective was to determine the boundary plane orientation relative to the adjacent grain crystal orientations. Trenches about $10 \mu \mathrm{m}$ in width with depth up to $10 \mu \mathrm{m}$ were cut across the selected grain boundaries using a FEI Nova NanoLab 600 Focused Ion Beam/Field Emission Gun (FIB/FEG) scanning electron microscope. Analysis of the exposed boundaries was done in combination with electron backscatter diffraction (EBSD) analysis of the sample surface, which was performed prior to FIB milling. The trace analysis method described in references $[16,17]$ was used to determine the angles $\alpha$ and $\beta$, which define the approximate grain boundary plane. The effect of sample tilt in the microscope on the apparent angles was corrected for. An example of such an analysis is shown in Figure 7.

The grain boundary planes were compared with the expected facet planes, with refererence to the lattices of the adjacent grains. Facetting in alumina is known to occur [18] on the basal (C) $(0001)$, rhombohedral (R) $\{01 \overline{1} 2\}$, prismatic (A) $\{11 \overline{2} 0\}$, Pyramidal (P) $\{11 \overline{2} 3\}$, and structural rhombohedral $(S)\{01 \overline{1} 1\}$ planes. The prismatic $(M)\{01 \overline{1} 0\}$ is also a low energy 
plane, but has not been observed to form facets. The analysis was done for seven boundaries at three locations (two locations A and B in the pure alumina, and at one location, $\mathrm{C}$, in the Cr-doped alumina). At each location there was a boundary associated with a strain feature, which it was concluded was cracked; the other boundaries were from adjacent grains that were exposed by the FIB trench. An example of a cracked boundary in the pure alumina sample is shown in Figure 8, in which the pole of grain boundary G12 (between grains $G 1$ and G2) is found to be close to that of the basal plane of grain $G 1$. The uncertainty of the pole orientations was approximately $2^{\circ}$, indicated by the dotted circle.

The data are summarised in Table 1 for the pure alumina and $\mathrm{Cr}$-doped alumina. Some of the grain boundary planes are found to be close to facet planes (i.e. boundaries $\mathrm{G} 12$ and G34 at location A and boundaries G12, G23 and G34 at location C). A recent threedimensional observation of a larger population of grain boundary planes in a coarse grain size alumina by diffraction contrast tomography [19] also observed a preference for these facet planes, although many boundaries had more general plane orientations. That is consistent with these observations. Although several boundaries were observed to be close to facet planes, there is no clear correlation between the incidence of facetted boundaries and cracking, and similarly no clear correlation with cracking and the relative crystal misorientation between the grains; it has been proposed that high crystallographic misorientations between the (0001) poles may caused significant thermal stress due to the anisotropy of thermal expansion coefficient in the alumina crystal [20].

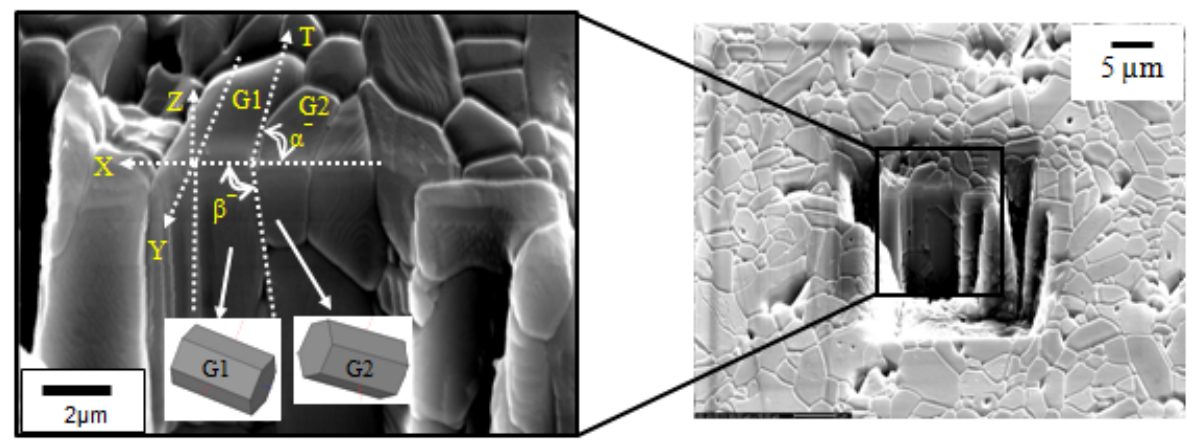

a)

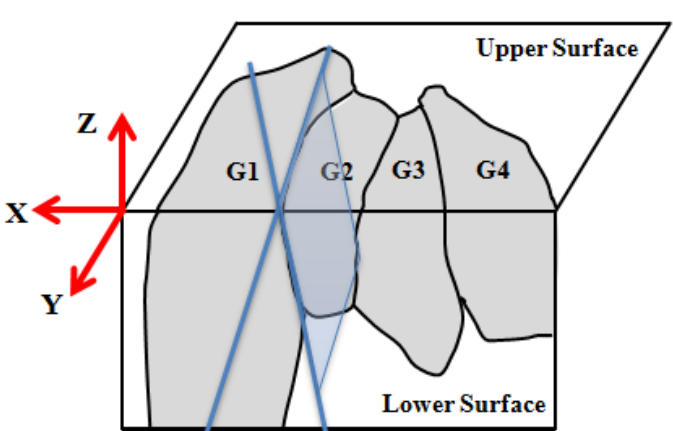

b)

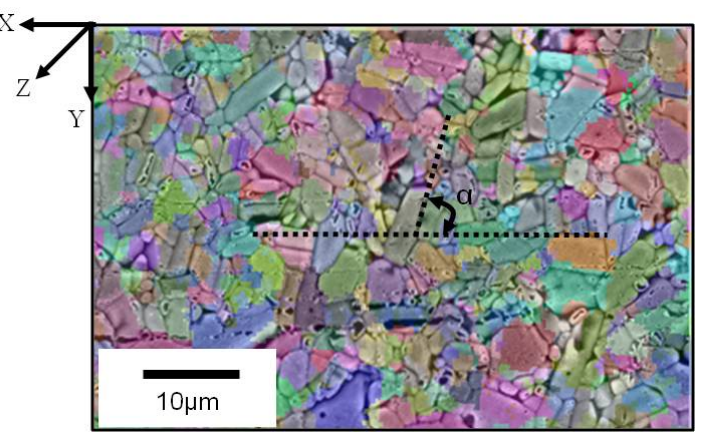

c)

Figure 7: Trace analysis of grain boundary planes for a set of four adjacent grains (G1 to G4): a) Trace (T) of the grain boundary, G12, between G1 and G2 on the orthogonal surfaces after FIB trench cutting, b) Schematic diagram, showing the traces of the grain boundary between G1 and G2 on orthogonal surfaces (upper and lower). c) EBSD map overlaid on the SEM image of the microstructure to show the surface trace of $\mathrm{G} 12$, and the trench orientation, parallel to the $\mathrm{X}$ direction. 


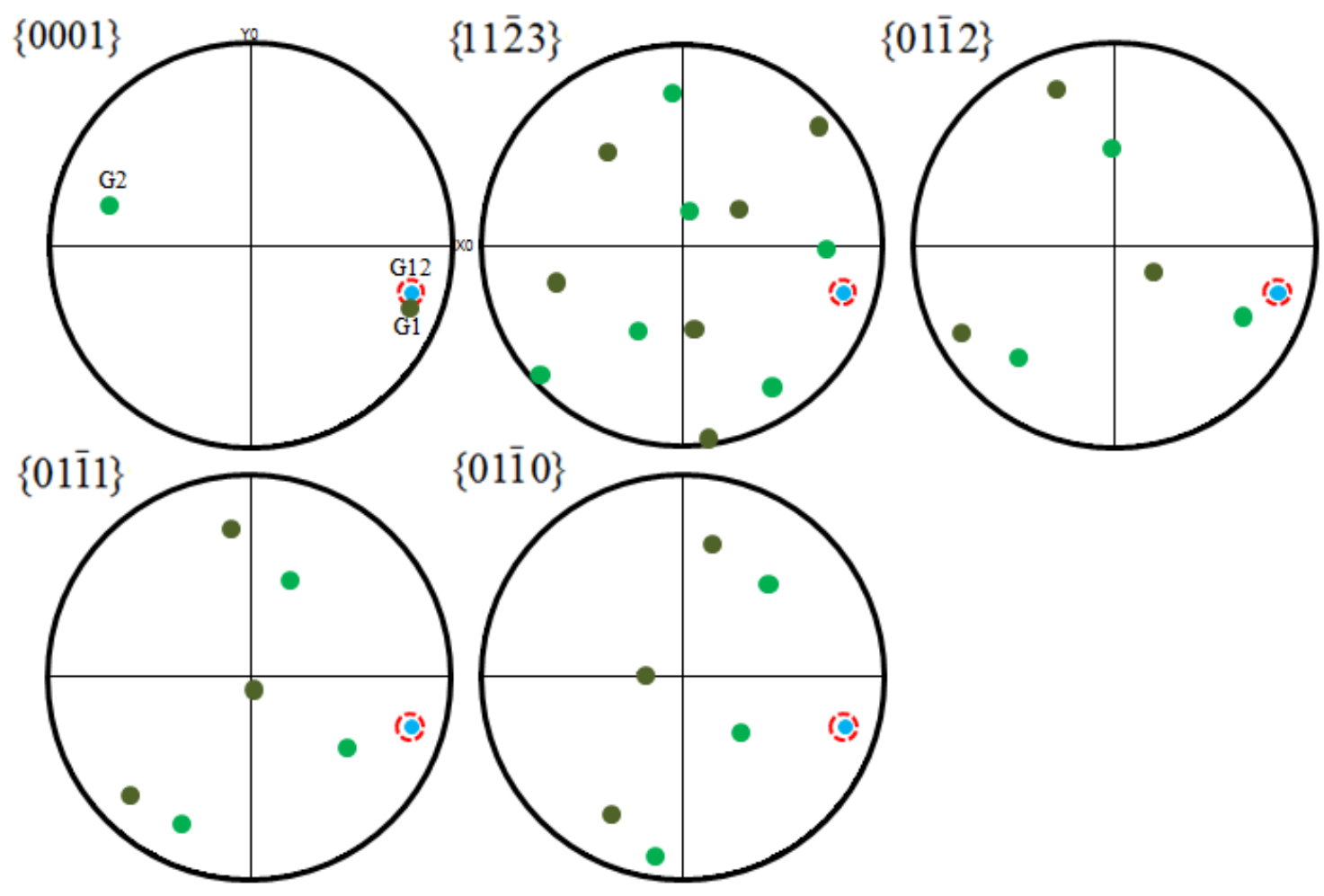

Figure 8: Pole figures for the grain boundary plane, $\mathrm{G} 12$, of the common facet planes in alumina in grains $\mathrm{G} 1$ (dark) and G2 (light). The boundary plane is close to the (0001) facet plane in G1.

The FIB sectioning of cracked boundaries allows the effect of the grain boundary plane to be addressed in the estimation of intergranular stresses. The relative stresses that develop at a grain boundary were estimated by considering the requirement to accommodate the net dimensional change between adjacent grains that arises on cooling from the sintering temperature (typically $1680^{\circ} \mathrm{C}$ ); the thermal expansion coefficient perpendicular to the grain boundary plane was calculated for each grain, assuming an elliptical variation with orientation between the maximal and minimal values (i.e. perpendicular and parallel to the basal plane) of $9.38 \times 10^{-6}$ and $8.62 \times 10^{-6} \mathrm{~K}^{-1}$ respectively, (data taken from [20]). A neighbourhood of many grains of varying orientation surrounds each grain; hence the relative displacement between the centres of adjacent grains is determined by the bulk thermal expansion coefficient, which was taken to be an average crystal thermal expansion coefficient $\left(9.00 \times 10^{-6} \mathrm{~K}^{-1}\right)$. The relative intergranular displacement perpendicular to the grain boundary was calculated from the difference between their relative centre displacements and the net dimensional change of the grains perpendicular to the boundary plane, $i$ e. subtracting the bulk dimensional change (average thermal expansion coefficient $x$ temperature change $x$ sum of grain dimensions) from the sum of the dimensional changes of adjacent grains (thermal expansion coefficient perpendicular to boundary $x$ temperature change $x$ grain dimension).

This analysis finds that the cracked boundaries (G12 in each case) have high values of net coefficient of thermal expansion (CTE) (i.e. the sum of the coefficients of thermal expansion perpendicular to the grain) (Figure 9a), and also of net thermal displacement (Figure 9b), though high values are also calculated at some other boundaries. If it is assumed that the displacement is accommodated by elastic strains over a distance that scales with the local grain size (e.g. $5 \%$ of the grain dimension perpendicular to the boundary), then the cracked boundaries tend to have the highest tensile stresses (Figure 9c). 
Table 1: Data for the oberved relationships between grain orientations and observations of cracking for pure alumina ( $A$ and $B$ ) and $\mathrm{Cr}$-doped aluma $(C)$ : the angles between the grain boundary poles and the pole for the facet planes and the misorientation between the basal poles of adjacent grains are given.

\begin{tabular}{|c|c|c|c|c|c|c|c|c|c|c|}
\hline \multirow{2}{*}{ Location } & \multirow{2}{*}{\multicolumn{2}{|c|}{$\begin{array}{l}\text { Grain } \\
\text { Boundary } \\
\text { \& Grains }\end{array}$}} & \multirow{2}{*}{$\begin{array}{l}\text { Grain } \\
\text { Size } \\
(\mu \mathrm{m})\end{array}$} & \multirow{2}{*}{$\begin{array}{c}\text { Angle }\left({ }^{\circ}\right) \\
\text { between } \\
(0001) \\
\text { poles }\end{array}$} & \multicolumn{5}{|c|}{$\begin{array}{l}\text { Angle }\left({ }^{\circ}\right) \text { between the grain boundary pole } \\
\text { and the nearest crystallographic pole }\end{array}$} & \multirow{2}{*}{ Observation } \\
\hline & & & & & (0001) & $\{11 \overline{2} 3\}$ & $\{01 \overline{1} 2\}$ & $\{01 \overline{1} 1\}$ & $\{01 \overline{1} 0\}$ & \\
\hline \multirow{6}{*}{$A$} & \multirow{2}{*}{ G12 } & G1 & 2.34 & \multirow[b]{2}{*}{138} & 4 & 40 & 49 & 63 & 66 & \multirow{2}{*}{ Cracked } \\
\hline & & G2 & 1.89 & & 18 & 26 & 19 & 34 & 51 & \\
\hline & \multirow{2}{*}{ G23 } & $\mathrm{G} 2$ & 1.89 & \multirow[b]{2}{*}{28} & 20 & 26 & 21 & 37 & 53 & \multirow[b]{2}{*}{ Not cracked } \\
\hline & & G3 & 1.95 & & 8 & 53 & 52 & 64 & 81 & \\
\hline & \multirow{2}{*}{ G34 } & G3 & 1.67 & \multirow{2}{*}{60} & 12 & 52 & 48 & 61 & 79 & \multirow{2}{*}{ Not cracked } \\
\hline & & G4 & 2.00 & & 61 & 22 & 4 & 8 & 25 & \\
\hline \multirow{8}{*}{ B } & \multirow{2}{*}{ G12 } & G1 & 3.25 & \multirow{2}{*}{116} & 26 & 18 & 32 & 41 & 56 & \multirow{2}{*}{ Cracked } \\
\hline & & $\mathrm{G} 2$ & 3.29 & & 36 & 31 & 44 & 55 & 78 & \\
\hline & \multirow{2}{*}{ G23 } & $\mathrm{G} 2$ & 2.98 & \multirow{2}{*}{117} & 17 & 34 & 34 & 47 & 67 & \multirow{2}{*}{ Not cracked } \\
\hline & & G3 & 0.69 & & 52 & 17 & 40 & 47 & 55 & \\
\hline & \multirow{2}{*}{ G34 } & G3 & 0.79 & \multirow{2}{*}{77} & 52 & 33 & 41 & 47 & 37 & \multirow{2}{*}{ Not cracked } \\
\hline & & G4 & 0.69 & & 43 & 18 & 20 & 34 & 48 & \\
\hline & \multirow{2}{*}{ G45 } & G4 & 0.96 & \multirow{2}{*}{99} & 41 & 15 & 19 & 34 & 47 & \multirow{2}{*}{ Not cracked } \\
\hline & & G5 & 2.18 & & 77 & 18 & 29 & 22 & 19 & \\
\hline \multirow{6}{*}{ C } & \multirow{2}{*}{ G12 } & G1 & 1.2 & \multirow{2}{*}{60} & 10 & 24 & 61 & 60 & 43 & \multirow{2}{*}{ Cracked } \\
\hline & & $\mathrm{G} 2$ & 6.5 & & 64 & 6 & 31 & 24 & 31 & \\
\hline & $G 23$ & G2 & 6.5 & 24 & 55 & 27 & 41 & 32 & 28 & Not cracked \\
\hline & G23 & G3 & 2.1 & 24 & 46 & 23 & 8 & 6 & 22 & Not cracked \\
\hline & $G 34$ & G3 & 2.1 & 66 & 45 & 13 & 33 & 36 & 27 & Not Cracked \\
\hline & G34 & G4 & 4.5 & 60 & 63 & 8 & 18 & 3 & 15 & Not Cracked \\
\hline
\end{tabular}

This simple model predicts that a larger thermal expansion coefficient and grain dimension perpendicular to the grain boundary encourage high tensile intergranular stresses, i.e. larger grains with facets close to the basal plane should develop higher tensile stress than other boundaries, particularly when both grains are so oriented. The predicted effect of grain boundary plane is generally supported by the limited observations of cracking detected by digital image correlation; the cracked boundaries are associated with high predicted tensile stress, but the model is also clearly very crude.

Recently, a detailed, image-based, 3-dimensional finite element model of stress development in a polycrystalline structure of alumina [21] also showed a dependence of average intergranular stress on the relative orientation of the grain boundary plane to the basal plane of one or both of its neighbouring grains, similarly to the simple model presented here. Interestingly, the 3D model [21] did not show any sensitivity of the average grain boundary stress to the relative grain misorientations, nor did it identify an effect of grain dimension. However, the predicted stresses were quite scattered due to the interaction of multiple grains, and such effects may be of secondary importance compared to grain boundary plane orientation. The interactions of multiple grains also introduce additional stresses, which may influence the propensity for crack nucleation. 

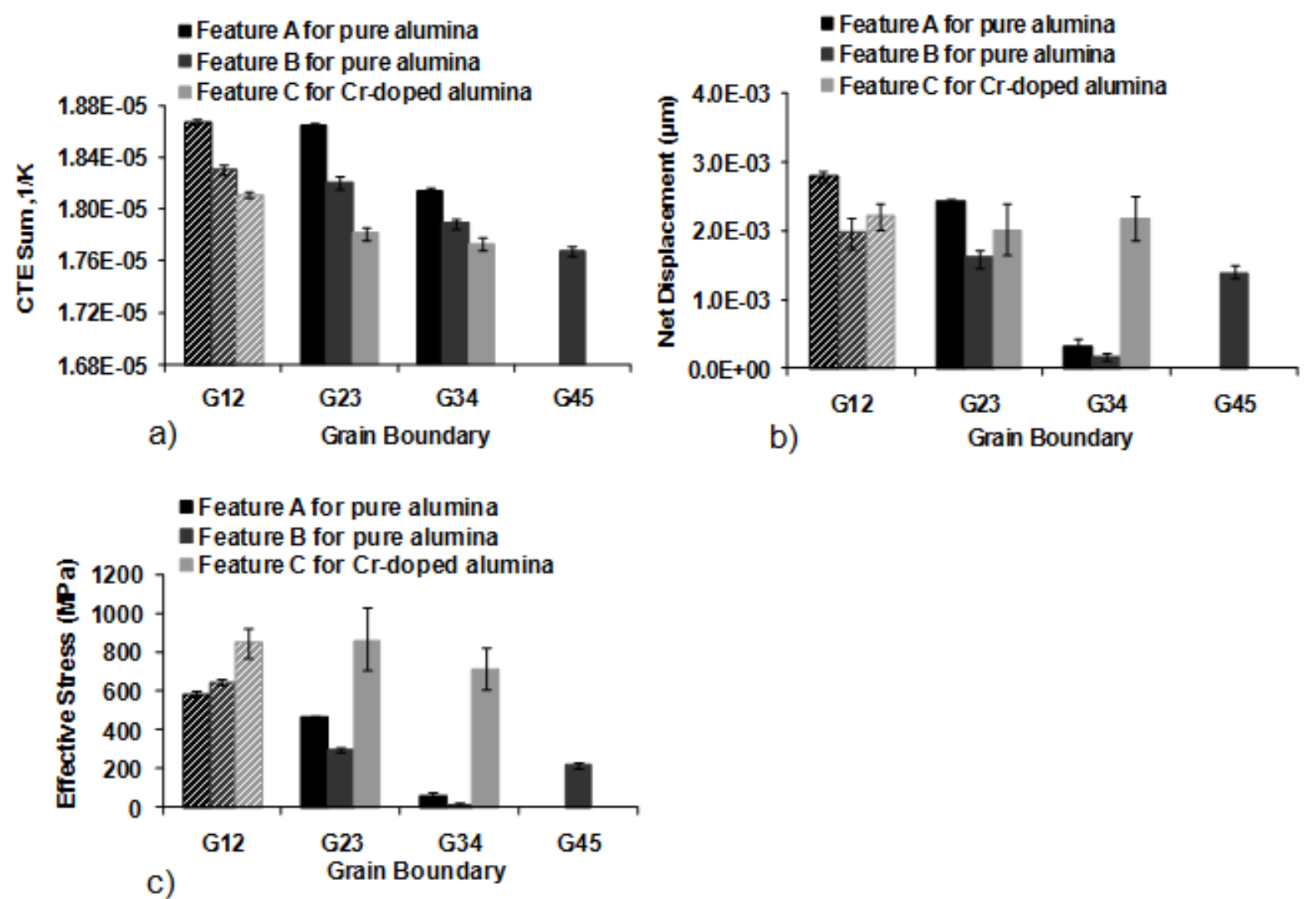

Figure 9: Estimation of intergranular thermal stresses using a simple model, a) net thermal expansion coefficient, b) net thermal displacement perpendicular to the boundary, c) thermal stress at boundary (The elastic properties of alumina are essentially isotropic [20], with a modulus of $498 \mathrm{GPa}$ ). (Cross hatching shows the cracked boundaries).

In summary, digital image correlation has enabled the identification of stable intergranular crack nuclei for the first time, which could then be characterised by electron-microscopy to determine the local microstructure and grain orientations. The limited observations presented here suggest that grain boundary facets close to the basal plane may develop higher tensile thermal stresses, which will encourage intergranular crack nucleation in polycrystalline alumina. The population of such boundaries, and their size, would therefore affect the flexural strength in the absence of other significant defects. Additions of silica to alumina sintered at $1400^{\circ} \mathrm{C}$ have been observed to increase the abundance of facet boundaries close to the basal plane [22]. Optimisation of microstructure, through control of the grain boundary population through sintering [23] or dopants might therefore have the potential to improve mechanical properties of polycrystalline aluminas. The techniques demonstrated here may be used to verify the relationship between grain boundary structure, neighbourhood and propensity for cracking.

\section{CONCLUSIONS}

Digital image correlation of optical images can be used to detect crack nuclei in fine grain size alumina. Such cracks would otherwise be non-visible; their detection allows characterisation of the grain boundary plane and grain orientations at crack grain boundaries.

Highest tensile stresses are predicted at the boundaries of larger grains with basal plane facets, and the locations of the crack nuclei are generally consistent with this simple model for intergranular thermal strains. 


\section{ACKNOWLEDGEMENTS}

The authors gratefully acknowledge the Ministry of Higher Education and scientific Research in Iraq for financial support and the Almath Crucibles Ltd, Newmarket, UK for providing the alumina samples.

\section{REFERENCES}

1. J. Seidel, N. Claussen, J. Rodel, Reliability of Alumina Ceramics - Effect of GrainSize. Journal of The European Ceramic Society, 1995. vol.15, no.5: pp. 395-404.

2. R. W. Rice, R. C. Pohanka, Grain-Size Dependence of Spontaneous Cracking In Ceramics. Journal of The American Ceramic Society, 1979. vol.62, no. (11-1): pp. 559-563.

3. A. G. Evans, Dimensional Analysis of The Grain-Size Dependence of Strength. Journal of The American Ceramic Society, 1980. vol. 63, no. (1-2): pp. 115-116.

4. M. A. Sutton, et al., Determination of displacements using an improved digital correlation method. Image and Vision Computing. 1983. vol.1, no.3: pp. 133-139.

5. S. P. McKenna, W. R. McGillis, Performance of digital image velocimetry processing techniques. Experiments in Fluids. 2002. vol. 32, no1: pp. 106-115.

6. J.Q. Fonseca, P.M. Mummery, P.J. Withers, Full-field strain mapping by optical correlation of micrographs acquired during deformation. Journal Of MicroscopyOxford, 2005. vol.218: pp. 9-21.

7. LaVision, DaVis Strain MasterMaster Softerware Manual 7.1. LaVision GmbH, Gottingen, 2006.

8. T.J. Marrow, G. A. D. Briggs, S.G.Roberts. In-situ scanning acoustic microscopy of crack bridging in alumina. Journal of the European Ceramic Society, (1994). vol.14: pp. 111-116.

9. S. Rahimi, D.L.Engelberg., J.A. Duff and T.J. Marrow., In-situ Observation of Intergranular Crack Nucleation in a Grain Boundary Controlled Austenitic Stainless Steel. Journal of Microscopy, (2009). vol.233: pp. 423-431.

10. P. M. Wood, J. A. Duff., T. J. Marrow, Imaging Autoclave Development for In-Situ Optical Measurement of High Temperature Aqueous Corrosion Processes. 12th International Conference on Fracture, ICF12, 12-17 July, Ottawa, Canada, (2009).

11. A. Cook, J.Duff., N. Stevens, S. Lyon, A. Sherry and T. J. Marrow, Preliminary Evaluation of Digital Image Correlation for In-situ Observation of Low Temperature Atmospheric-Induced Chloride Stress Corrosion Cracking in Austenitic Stainless Steels. 216th ECS (Electrochemical Society) Meeting, 4-9 Oct, Vienna, 2009.

12. H. Li, J.Duff andT.J.Marrow, In-situ observation of crack nucleation in nuclear graphite by digital image correlation. Proceedings of PVP2008 ASME Pressure Vessels and Piping Division. (2008). Conference, July 27-31, 2008, Chicago, Illinois, USA. Paper PVP2008-61136. 
13. M. Mostafavi, J.Duff., R.Delorme, T.J. Marrow, In-situ Observation of Damage Nucleation in Nuclear Graphite under Biaxial Flexural Loading. ECF18, Dresden, June 2010.

14. Y. M. Wong, et al., Automation and dynamic characterization of light intensity with applications to tapered plastic optical fibre. Journal of Optics A-Pure And Applied Optics, 2003. vol. 5, no.4: pp. S51-S58.

15. P. D. Warren, D. A. Hills, and S.G. Roberts, Surface Flaw Distributions in Brittle Materials and Hertzian Fracture. Journal of Materials Research, 1994. vol.9. no.12: pp. 3194-3202.

16. V. Randle, D. Dingley, Measurement of Boundary Plane Inclination In A Scanning Electron-Microscope. Scripta Metallurgica, 1989. 23(9): p. 1565-1569.

17. V. Randle, Crystallographic Characterization of Planes In The Scanning ElectronMicroscope.Journal of the Materials Characterization, 1995. vol.34, no.1: pp. 29-34.

18. J. H. Choi, et al., Equilibrium shape of internal cavities in sapphire. Journal Of The American Ceramic Society, 1997. vol.80, no.1: pp. 62-68.

19. T.J. Marrow, A.K., P. Reischig, S. Rolland du Roscoat, W Ludwig, Three-dimensional Tomography of Alumina. Sosman Memorial Award symposium, MS\&T 2009, Pittsburgh, PA.

20. S. G. Yousef, J. Rodel.E. R. Fuller, A. Zimmermann, B. S. .EL-Dasher Microcrack Evolution in Alumina Ceramic: Experimental and Simulation. Journal of American Ceramic Society, 2005. vol.88, no.10. pp.2809-2816.

21. T.J. Marrow, M. A. Aswad, J.Q. Fonseca, and P.J. Withers, In-situ observation and modelling of intergranular cracking in polycrystalline alumina. Sixth international conference on Materials Structure \& Micromechanics of Fracture (MSMF6), Brno, Czech Republic, June 28 - 30, 2010.

22. M. J. Kim, S. M. Kim, and D. Y. Yoon, "Singular Grain Boundaries in Alumina Doped with Silica," Journal of the American Ceramic Society, vol.87, no.3, pp. 507-509, 2004.

23. S. J. Dillon, H. Miller, M. P. Harmer, and G. S. Rohrer, "Grain boundary plane distributions in aluminas evolving by normal and abnormal grain growth and displaying different complexions," International Journal of Materials Research, vol.101, no.1, pp. 50-56, 2010.

Corresponding author: james.marrow@materials.ox.ac.uk 